\title{
Antiretroviral Therapy and HIV-Associated Neurocognitive Disorders
}

\author{
Cagla Akay ${ }^{1}$ and Kelly L. Jordan-Sciutto ${ }^{2}$ \\ ${ }^{1}$ Department of Pathology, University of Pennsylvania \\ 2Department of Pathology, University of Pennsylvania \\ United States of America
}

\section{Introduction}

Human immunodeficiency virus (HIV)-Associated Neurocognitive Disorders (HAND) define a wide spectrum of behavioral, cognitive and motor dysfunctions(Heaton, Clifford et al. ; Neuenburg, Brodt et al. 2002; McArthur 2004; Lindl, Marks et al. 2010). The initial criteria put forth by the American Association of Neurology described two levels of neurocognitive impairment in HIV $(+)$ patients: HIV-associated dementia (HAD) and minor motor cognitive disorder (MCMD)(1996). However, the emergence of more subtle forms of cognitive impairment after the implementation of combination antiretroviral therapy (cART) in 1996 necessitated a revised classification, which now includes, in addition to HAD, asymptomatic neurocognitive impairment (ANI), and mild neurocognitive disorder (MND), while MCMD is no longer included in the classification (Antinori, Arendt et al. 2007). While the incidence of HAD has declined in the cART era, from $10-15 \%$ to approximately 2\%; ANI and MND have become more prevalent (Heaton, Clifford et al. 2010). Overall, HAND continues to persist, affecting approximately $50 \%$ of $\mathrm{HIV}(+)$ patients in the cART era (Power, Boisse et al. 2009; Heaton, Clifford et al. 2010). The underlying neuropathology suggests a change from overt dementia to a more insidious neurocognitive impairment (Kusdra, McGuire et al. 2002; Anthony and Bell 2008; Everall, Vaida et al. 2009). Various factors, such as age-related vascular and metabolic changes and substance and alcohol abuse, the emergence of resistant virus species, persistent viral DNA in the central nervous system (CNS) despite successful plasma viral control, limited access of cART into the CNS, poor adherence to drug regimen, and possible cART-related neurotoxicities are suggested to contribute to these changes observed in the clinical and neuropathological presentation in the HAND brain. With the increased life expectancy, the ever-expanding repertoire of antiretroviral drugs (ARVs) and the patient-tailored, dynamic combination ARV regimens, the contribution of CART to the persistence of HAND is an urgent question that needs to be addressed to more successfully predict and prevent CNS related morbidity and mortality among HIV-infected patients.

\section{Clinical presentation of HAND}

HAND was initially characterized as a combination of acquired deficits in cognition and motor function, in addition to behavioral and emotional changes (Navia, Cho et al. 1986; 
Portegies, Enting et al. 1993). When it became apparent that viral replication could not be controlled by the use of one or two antiretroviral drugs, and that the CNS was shown to provide a sanctuary for HIV (Brew, Pemberton et al. 1997; Pialoux, Fournier et al. 1997), more aggressive treatment plans employing multiple ARVs were implemented. Successful viral suppression due to cART led to the observed initial improvement in cognitive function and an overall decrease in the incidence of the most severe form of HAND, HAD. However, as patients are maintained on CART long-term, subtler forms of neurocognitive impairment, ANI and MND have since emerged (Power, Boisse et al. 2009; Heaton, Clifford et al. 2010). ANI is defined as mild neurocognitive impairment in the absence of a decline in activities of daily living (ADL), whereas mild-to moderate neurocognitive decline that affects ADL is considered MND. The areas of cognition affected include concentration and/or attention, executive functioning, memory, and visuospatial skills (Antinori, Arendt et al. 2007). Depression, psychosis and anxiety at varying severity are also commonly observed in HIVinfected patients (Owe-Larsson, Sall et al. 2009). The complexity of cognitive and behavioral presentations in CART treated patients has served to obfuscate the underlying causes of these changes.

The clinical course of disease progression of HAND has changed dramatically since the introduction of cART in 1996. In the pre-cART era, HIV-associated neurocognitive impairment tended to worsen over time, and was more likely to follow a fulminant course. In fact, when other risk factors were controlled for, HAD was associated with increased mortality (Ellis, Deutsch et al. 1997). In contrast, the neurocognitive symptoms associated with HIV appear to follow a more unpredictable course during the life-time of the patient in the cART era. While some patients may recover from MND with effective cART, others can worsen steadily with progression from ANI to HAD. Further yet, some patients might experience a fluctuating course (Cole, Margolick et al. 2007; Woods, Moore et al. 2009). However, while progression to HAD is still observed in a subset of patients, even though at a slower pace in most cases, the mortality is now less likely to associate with HAND, and more likely with co-existing factors, such as co-infections, substance and alcohol use, and age-related illnesses.

\section{Neuropathology of HAND}

HIV targets and leads to a selective loss of $\mathrm{CD}^{+} \mathrm{T}$ cells, which eventually causes the severe immunodeficiency that develops in cases of uncontrolled HIV infection. HIV also targets monocytes and macrophages early during infection, which enable HIV to establish viral reservoirs in the lymphoid tissue and the CNS (Gendelman, Lipton et al. 1994; GonzalezScarano and Martin-Garcia 2005; Kaul, Zheng et al. 2005). In fact, extensive in vitro and in vivo studies support the "Trojan Horse" model of HIV infection in the CNS (Liu, Lossinsky et al. 2002). According to this model, HIV enters the brain via infected monocytes that cross the blood-brain barrier (BBB). Once in the CNS, infected monocytes differentiate into macrophages, which then constitute viral reservoirs in the brain. A small number of neurons and glia may also become infected; however, such infection is not productive (Gendelman, Lipton et al. 1994; Gonzalez-Scarano and Martin-Garcia 2005; Kaul, Zheng et al. 2005).

Two major mechanisms of neuronal damage have been proposed: 1) Direct neurotoxicity by viral proteins such as HIV envelope glycoprotein (gp120) and HIV transactivator of transcription (Tat) protein, and, 2) Indirect neurotoxicity induced by soluble factors released by infected and/or activated macrophages including, but not limited to, quinolinic acid, 
TNF- $\alpha$, reactive oxygen species (ROS), and cytokines such as CXCL12 (stromal cell-derived factor-1, SDF-1), CCL2 (monocyte chemotactic protein-1, MCP-1), and interleukin-6 (IL6)(Price, Brew et al. 1988; Giulian, Yu et al. 1996; Soontornniyomkij, Nieto-Rodriguez et al. 1998; Lindl, Marks et al. 2010). Most of these factors not only directly affect neurons but also induce the secretion of more of these and other neurotoxic soluble factors, such as excitatory amino acids such as glutamate from neighboring macrophages/microglia, and astrocytes (Gorry, Ong et al. 2003; Gonzalez-Scarano and Martin-Garcia 2005). In the chronic course of HIV infection, inflammatory events and oxidative stress that ensue in this environment in the CNS precipitate neuronal dysfunction and death. Several in vitro and in vivo studies lend support to the indirect mechanism of neurotoxicity. Increases in neuroinflammatory factors (e.g. TNF- $\alpha$, CCL2, and IL-6) have been detected in the cerebrospinal fluid (CSF) of HIVinfected patients (Sippy, Hofman et al. 1995; Achim, Masliah et al. 1996; Achim and Wiley 1996; Conant, Garzino-Demo et al. 1998; Gisolf, van Praag et al. 2000). Further, markers of oxidative stress, such as 4-hydroxynonenal (4-HNE), are well documented in HAND patients (Haughey, Cutler et al. 2004; Sacktor, Haughey et al. 2004). In addition, several studies have reported that neopterin and $\beta 2$-microglobulin, regarded as indirect markers of macrophage activation, are elevated in the CSF of HIV infected patients (Yilmaz, Fuchs et al. 2006; Edén, Fuchs et al. 2010; Hagberg, Cinque et al. 2010). Finally, the neuropathological correlates of inflammation, including astrocytosis and increased perivascular macrophage infiltration are commonly observed in the brains of HIV-infected patients (Kolson 2002; Kusdra, McGuire et al. 2002; Lindl, Marks et al. 2010).

The precise mechanisms by which inflammation leads to neuronal death in response to these functionally diverse factors are not known; however, several mechanisms are likely candidates. Intracellular ROS accumulation can directly alter neuronal mitochondrial function, leading to neuronal damage and death (Reynolds, Laurie et al. 2007; Hu, Sheng et al. 2009). On the other hand, pro-inflammatory factors can impair glutamate reuptake by astrocytes (Gorry, Ong et al. 2003; Gonzalez-Scarano and Martin-Garcia 2005). Increased extracellular glutamate that is not buffered by astrocytes can activate $N$-methyl-D-aspartate glutamate (NMDA) receptors and lead to excess $\mathrm{Ca}^{2+}$ influx into the neurons (Giulian, Yu et al. 1996; O'Donnell, Agrawal et al. 2006). Much evidence indicates that increased intracellular $\mathrm{Ca}^{2+}$ concentrations are detrimental to neurons. $\mathrm{Ca}^{2+}$ can precipitate oxidative stress via free radical production, to which neurons are likely to become more susceptible in the absence of normal functioning astrocytes and macrophages. Further, excess intracellular $\mathrm{Ca}^{2+}$ activates the death-associated proteases, calpains and caspases (Moore, Rothwell et al. 2002; Danial and Korsmeyer 2004). The pathological indicators of neuronal damage and death, dendritic simplification, axonal damage and synaptic loss, correlate best with the presence of infected macrophages and microglia (Masliah, Heaton et al. 1997; Ellis 2010). Interestingly, viral load (VL) does not correlate with neurodegeneration, suggesting that the indirect mechanisms might play a more important role in neuronal injury than the direct mechanisms (Tozzi, Balestra et al. 2007; Yilmaz, Price et al. 2008).

Several CSF markers have been utilized in an attempt to predict, diagnose and monitor the progression of HAND. Among those, as markers of immune activation, TNF- $\alpha$, and CCL2 levels were shown to correlate with neurological deficits in the pre-cART era (Gisolf, van Praag et al. 2000; Sevigny, Albert et al. 2004; Ragin, Wu et al. 2010); however, a more recent study did not show such a correlation for either of these markers among a cohort of patients on cART (McArthur, McDermott et al. 2004). Additionally, contrary to expectations, in patients on CART regimens with high predicted CSF concentrations, the markers of 
inflammation and oxidative stress appear to persist (Roc, Ances et al. 2007). Further, while cART has been shown to decrease neopterin levels in the CSF, these levels still appear to be above those measured in uninfected individuals (Yilmaz, Fuchs et al. 2006). A recent study shows that the changes in CSF neopterin levels are not affected by ARVs with predicted high CSF concentrations (Eden and Fuchs 2010). These findings imply that neuroinflammation might be ongoing despite the success of cART in controlling viral replication; thus supplementary therapies that would alleviate inflammation appear necessary for a better control of HAND.

\section{HAND and Combination Antiretroviral Therapy}

The development of more sensitive reverse transcriptase polymerized chain reaction (RTPCR) methods for the detection of HIV RNA during the 1990s revealed that single ARV treatments were not able to completely suppress viral replication. It also became apparent that the development of ARV resistance was hampering the efforts to successfully suppress VL. These major concerns led to a revision in the treatment plan for HIV infection, which was initially called highly active antiretroviral therapy (HAART), and was later changed to combination antiretroviral therapy (cART). Currently recommended cART for ARV-naïve HIV-infected patients includes a cocktail of ARVs from three different classes, nucleoside/nucleotide reverse-transcriptase inhibitors (NRTIs/NtRTIs), non-nucleoside reverse-transcriptase inhibitors (nNRTIs), and protease inhibitors (PIs). Sustained viral suppression, improved immune function and better control of HIV-related co-morbidities have contributed to the reduction in HIV-associated morbidity and mortality in the era of cART.

In addition to its success in the periphery, cART led to striking changes in the clinical presentation and disease progression of HAND. The incidence of frank HAD has decreased dramatically and HAND in the cART era follows a more protracted course. However, neurocognitive impairment still persists, albeit as a general rule it is not as severe as in the pre-cART era. Importantly, HAD patients on cART still exhibit increased levels of macrophage infiltration similar to those seen in patients with HAD in the pre-cART era. Virus-related factors, such as persistent viral DNA in the CNS despite successful suppression of plasma and CSF VL and the emergence of resistant virus species due to failure of ARV to reach therapeutically relevant concentrations in the CSF, likely account at least partially for these observations.

Studies in animal models of HIV infection suggest that the seeding of HIV in the CNS can occur as early as 4 days after the initial exposure to the virus. In addition, HIV RNA can be detected in the human brain as early as 14 days after infection. These studies indicate that viral escape to the CNS during the initial phase of asymptomatic viremia is possible; thus early and sustained VL control is necessary to limit the establishment of an HIV reservoir in the CNS, which, as a consequence, would have an immediate impact on the development of HAND. However, the timing for cART initiation in ARV-naïve HIV-infected individuals is not straightforward due to serious systemic ARV-related side effects and potential non-adherence, which in itself carries increased risk for selection of drug-resistant virus strains. Based on data from large clinical trials revealing that the initial CD4 cell count is a major predictor of morbidity and mortality in HIV patients, The United States Panel on Antiretroviral Guidelines for Adults and Adolescents recommends that cART should be initiated in patients with CD4 cell counts lower than 350 cells/ $\mu$ l or in those with an AIDS-defining illness irrespective of 
CD4 counts (Panel on Antiretroviral Guidelines for Adults and Adolescents. (Revised January 10, 2011) Guidelines for the use of antiretroviral agents in HIV-1-infected adults and adolescents, April 14, 2011, available from http://www.aidsinfo.nih.gov/ContentFiles/ AdultandAdolescentGL.pdf). cART is also recommended for patients with CD4 counts between $350-500$ cells $/ \mu$ l. The panel also states that cART should be considered irrespective of the CD4 counts in patients who have either HIV-associated nephropathy, or Hepatitis B infection that needs treatment. For those who have CD4 counts above 500, the panel was evenly divided, with $50 \%$ of the panel members voted in favor of starting cART, whereas the other $50 \%$ of members voted cART treatment as optional under these conditions. However, the panel has not reached consensus with regard to the time to start CART in the context of HAND risk reduction, an important factor in the persistence of HAND in the cART era. Unfortunately, even with early initiation of cART, failure of ARVs to reach effective CSF concentrations may hamper the suppression of VL in the CNS. Thus in an attempt to assess the efficacy with which ARVs reach effective CSF concentrations, a scoring system coined CNS penetrance effectiveness (CPE) scoring is being increasingly investigated in clinical studies (Letendre, Marquie-Beck et al. 2008; Marra, Zhao et al. 2009; Tozzi, Balestra et al. 2009; Edén, Fuchs et al. 2010; Letendre, Ellis et al. 2010; Garvey, Winston et al. 2011; Smurzynski, Wu et al. 2011). An ARV drug with relatively poor CNS penetration is assigned a CPE score of 0 , while one with an intermediate CNS penetration would have a CPE score of 0.5 , and one with high penetration would receive a CPE score of 1 . Each combination ARV treatment regimen includes ARVs from at least three different classes with distinct physicochemical properties and thus the sum of CPE scores from several types of drugs will affect the functional capacity of the regimen within the CNS.

\section{Antiretroviral drugs}

While each ARV is unique in structure, each ARV class has certain common characteristics that aid in determining the overall CPE score of any given cART. However, the unique properties of each drug also make it harder to accurately predict the CPE score for that particular ARV, and by extension, CPE score for specific cART regimens as well. For a better understanding of an ideal cART regimen for the control of HAND, we need to examine each ARV class. ARVs target HIV at distinct steps in the life cycle of the virus, including (1) virus attachment to the host cell, both fusion with the cell membrane and entry, (2) reverse transcription, (3) integration, (4) processing of viral proteins, and (5) maturation. The major classes of antiretroviral drugs are entry inhibitors, nucleoside/nucleotide reverse transcriptase inhibitors (NRTIs), non-nucleoside reverse transcriptase inhibitors (nNRTIs), integrase inhibitors, and protease inhibitors (PIs). In this section, we will summarize the mechanisms of action, the general and CNS-specific side effects and the predictors of CPE scores for each class with the relevant in vitro and in vivo findings.

\subsection{Entry inhibitors}

HIV entry to the host cell begins with the binding of the HIV surface protein, gp120, to the T cell surface receptor CD4. This attachment exposes specific conserved regions of gp120, enabling its binding to one of the two chemokine receptors on the host cell surface, CXCR4 or CCR5. This, in turn, unmasks gp41, a process that leads to the fusion of the viral membrane with the host cell and the subsequent release of the virus capsid into the host cell cytoplasm. This highly conserved, stepwise process provides three access points which a 
relatively new class of ARVs, collectively termed entry inhibitors, targets. At this point, only two entry inhibitors are approved by the Food and Drug Administration (FDA) for clinical use: enfuvirtide (T-20), and maraviroc.

\subsubsection{Enfuvirtide}

Enfuvirtide is a 36 amino-acid synthetic peptide that mimics a specific region in gp41 and competitively inhibits the fusion step and does not depend on co-receptor use (Joly, Jidar et al. 2010). It is not a substrate for major drug metabolism enzymes, CYP3A4 or CYP2B6, a trait that limits possible interactions that might occur with other ARVs that are inhibitors or activators of these enzymes. Further, enfuvirtide acts in the extracellular area, and lacks cross-resistance. In addition, enfuvirtide-related side effects are minimal, and are usually limited to injection site reactions. Due to these characteristics, enfuvirtide is currently prescribed to ARV-experienced patients, especially those with multi-ARV resistance (Yeni, Mills et al. 2010). However, its potential benefits for patients with CNS involvement are not known. The only study where CNS penetrance of enfuvirtide was assessed in humans, has found CSF enfuvirtide levels to be below plasma $\mathrm{EC}_{50}$ (plasma concentration required for obtaining $50 \%$ of a maximum effect in vivo) values, which is most likely due to the high level of binding of enfuvirtide to plasma proteins (92\%), mainly albumin (Patel, Zhang et al. 2005; Price, Parham et al. 2008). CSF concentration of a given pharmaceutical does not reflect its parenchymal concentration with certainty, as will be discussed in section 6; however, as extracellular enfuvirtide concentration determines its antiviral activity; its CSF levels most likely reflect its concentrations in the parenchyma. Based on these data, enfuvirtide is given a CPE score of zero (table 1 for proposed CPE scores of currently used ARVs) (Smurzynski, Wu et al. 2011). Importantly, a limited number of studies have found no association between the use of enfuvirtide and peripheral neuropathy, a side effect associated with certain ARVs. These findings, in addition to its low CPE score, likely preclude any potential neurotoxicity issues in the CNS related to enfuvirtide. In summary, the impact of enfuvirtide on HAND, whether beneficial or toxic, is most likely minimal; however, its efficacy in controlling systemic VL will certainly contribute to better control of disease progression in the CNS.

\subsubsection{Maraviroc}

The second clinically approved member of the entry inhibitor family is maraviroc. Maraviroc is a specific, non-competitive CCR5 antagonist and is effective in both ARV-naïve and ARV-experienced patients who harbor a CCR5-tropic virus, which is determined by genotypic and phenotypic tests before the initiation of maraviroc-including regimens (Lieberman-Blum, Fung et al. 2008; Kromdijk, Huitema et al. 2010). It is a substrate for drug efflux proteins ((p-glycoprotein (p-gp) and multidrug resistance protein-1 (MDR-1)) expressed by the BBB; however, its binding affinity to plasma proteins is not very high. Thus, CSF and brain distribution studies in rats suggest that approximately $10 \%$ of the plasma maraviroc levels are achieved in the CNS, which falls within a range of reported $\mathrm{IC}_{50}$ (half maximal inhibitory concentration) values (Walker, Bowers et al. 2008). These predictions are further supported by multiple studies conducted in humans, where maraviroc achieves therapeutically relevant concentrations in the CSF, and also effectively suppresses CSF VL (table 1) (Smurzynski, Wu et al. 2011). Currently no data exists on the possible toxic effects of maraviroc in the CNS; however, given that the only cell types that express CCR5 are macrophages and T cells, direct neurotoxicity due to maraviroc is not likely. Thus, cART regimens that include maraviroc are increasingly prescribed and future studies are warranted to examine its impact on HAND. 


\begin{tabular}{|c|c|c|c|}
\hline Antiretroviral Class & 1 & CPE Score & 0.5 \\
\hline Entry Inhibitors & Maraviroc & & Enfuvirtide \\
\hline $\begin{array}{c}\text { Nucleoside/nucleotide } \\
\text { reverse transcriptase } \\
\text { inhibitors }\end{array}$ & Zidovudine (AZT) & Stavudine (d4T) & Tenofovir (TDF) * \\
\hline & Abacavir (ABC) & Lamivudine (3TC) & \\
\hline & & $\begin{array}{c}\text { Emtricitabine } \\
\text { (FTC) * }\end{array}$ & \\
\hline nNRTIs & Nevirapine (NVP) & Efavirenz (EFV) & \\
\hline Protease Inhibitors & Darunavir & Amprenavir & Nelfinavir \\
\hline & & Atazanavir & Ritonavir * \\
\hline & & Fosamprenavir & Saquinavir * \\
\hline Integrase Inhibitors & & Indinavir & \\
\hline
\end{tabular}

Table 1. CNS penetrance effectiveness (CPE) scores of antiretroviral drugs currently in clinical use. Higher CPE scores indicate better penetrance. * In vivo data suggests better CPE score

\subsection{Nucleoside/nucleotide reverse transcriptase inhibitors \\ 5.2.1 Mechanism}

The single-stranded HIV RNA, once in the host cell, is reverse transcribed by the viral reverse transcriptase (HIV-RT) into a double-stranded complementary viral DNA, which migrates to the host nucleus as part of the pre-integration complex. Nucleoside and nucleotide reverse transcriptase inhibitors (NRTIs/NtRTIs) are analogues of the naturally occurring deoxynucleotides that are needed for the reverse-transcription (Warnke, Barreto et al. 2007); however, NRTIs/NtRTIs lack a 3'-hydroxyl group on the deoxyribose moiety. Three steps of phosphorylation are needed for NRTIs to become activated, while only two phosphorylation steps are necessary in the case of NtRTIs (Piliero 2004). Following activation, NRTIs/NtRTIs are incorporated into the viral DNA, thereby preventing the next $5^{\prime}-3^{\prime}$ phosphodiester bond formation and consequently leading to the early termination of viral DNA synthesis, named chain termination (Warnke, Barreto et al. 2007).

\subsubsection{Clinical use}

NRTIs/NtRTIs quickly and effectively decrease VL and increase CD4 counts (Hill and Sawyer 2009). As they are not metabolized by drug metabolizing enzymes, the activities of which are altered by certain ARV drugs from other classes, dose adjustment is usually not necessary. Currently, five NRTIs (zidovudine (AZT), stavudine (d4T), lamivudine (3TC), emtricitabine (FTC), abacavir (ABC)) and one NtRTI (tenofovir disoproxil fumarate (TDF)) are used and form the backbone of most initial cART regimens. The current initial cART regimen typically includes two NRTIs/NtRTIs in addition to an nNRTI or a PI boosted with ritonavir, which is also a PI. The recently revised recommendations suggest that initial cART in ARV-naïve patients should include one of the following cocktails: efavirenz/tenofovir/emtricitabine (EFV/TDF/FTC), ritonavir-boosted 
atazanavir/tenofovir/emtricitabine

$(\mathrm{ATV}+\mathrm{r} / \mathrm{TDF} / \mathrm{FTC})$,

ritonavir-boosted

darunavir/tenofovir/emtricitabine

$(\mathrm{DRV}+\mathrm{r} / \mathrm{TDF} / \mathrm{FTC}), \quad$ or

raltegravir

tenofovir/emtricitabine (RAL + TDF/FTC). A similar cART regimen is also recommended for post-exposure prophylaxis (Grant 2010). In addition, TDF/FTC cocktail is recommended as a pre-exposure prophylaxis regimen in high risk persons (Grant 2010). Further, combinations that include $\mathrm{AZT}, 3 \mathrm{TC}$, and $\mathrm{ABC}$ are proven to be the most effective in prevention of intrauterine and postnatal transmission, with relatively few side effects to both mother and child, when compared with regimens that include nNRTIs or PIs (Sturt, Dokubo et al. 2010). However, specific NRTIs/NtRTIs are not considered part of a first line therapy in certain patient populations. For example, in pregnant women TDF is only considered when the infecting virus shows resistance to other NRTIs or in the presence of chronic hepatitis infection. This is due to concerns regarding possible toxicity of TDF for the fetus, specifically in bone mineralization (Duarte-Rojo and Heathcote 2010). Similarly, d4T can lead to sometimes fatal lactic acidosis and hepatic failure in pregnant women and is only used when no other viable options remain (Nolan and Mallal 2004).

\subsubsection{Side effects}

Due to the low fidelity of HIV-RT and to the high rate of HIV replication, NRTI/NtRTIresistant viral strains have developed. The emergence of these strains along with the severe NRTI/NtRTI-associated side effects, including lactic acidosis, lipoatrophy, and peripheral neuropathy, usually necessitates changes in the regimen, which can include switching ARVs, adding more ARVs, and/or adjusting dosing (Kohler and Lewis 2007). Interestingly, individual NRTIs/NtRTIs are associated with specific side effects. For example, d4T, and to a lesser extent, AZT, are associated with lipoatrophy, while TDF does not appear to cause this particular side effect. Peripheral neuropathy, which manifests in approximately $40 \%$ patients on cART, is associated with regimens that include $\mathrm{d} 4 \mathrm{~T}$ (Kohler and Lewis 2007). On the other hand, TDF is associated with increased risk of nephropathy and loss of bone density (Duarte-Rojo and Heathcote 2010). FTC-associated side effects are usually milder than those observed with other NRTIs/NtRTIs and include skin discoloration, nausea, and elevated liver enzymes (Nelson and Schiavone 2004).

One major mechanism underlying NRTI/NtRTI-associated side effects is mitochondrial toxicity, which is a result of DNA polymerase $\gamma$ inhibition, leading to mitochondrial DNA depletion, oxidative stress, loss of mitochondrial membrane potential $(\Delta \Psi \mathrm{m})$, and loss of oxidative phosphorylation (Dalakas 2001; Dagan, Sable et al. 2002; William 2003; Nolan and Mallal 2004). The potency of NRTIs in inhibiting DNA polymerase $\gamma$ differs among the members, with d4T and AZT showing higher potency compared with other NRTIs/NtRTIs. Further, the activities of the different kinases responsible for phosphorylation of each type of NRTI/NtRTI show variability among different cell types and may account for the cell-type specificity of the side effects (Bazzoli, Jullien et al. 2010).

\subsubsection{Considerations for the CNS}

Based on extensive data compiled from animal and human studies, AZT, d4T, and ABC appear to reach effective concentrations in the CNS, while the access of 3TC to the CNS is predicted to be minimal (Anderson and Rower 2010). There is limited information on TDF distribution in the CNS; the only functional study investigating TDF access to the CNS suggests that its concentrations in the CNS might be limited, with varying degrees of 
accumulation in different brain regions (Anthonypillai, Gibbs et al. 2006). Currently, there is no information on CSF levels of FTC in the CSF (table 1) (Smurzynski, Wu et al. 2011). However, several reports suggest that regimens containing TDF and FTC may lead to improvements in neurocognitive impairment when compared with regimens that include other NRTIs or nNRTI/PIs (Allavena, Le Moal et al. 2006; Winston, Duncombe et al. 2010). Overall, clinical data from extensive studies show that NRTIs/NtRTIs, as part of cART regimens contribute significantly to the sharp decreases observed in the incidence of HAND. However, with regard to NRTIs/NtRTIs, several factors should be considered as possible contributors to the persistence of HAND. First, the emergence of NRTI/NtRTI resistance and possible less-than effective CSF concentrations, especially of 3TC and TDF, may limit their effectiveness in the eradication of the viral reservoirs in the CNS. In addition, mitochondrial toxicity due to NRTIs/NtRTIs may play a role in neuronal damage and death, as there is preliminary evidence that NRTIs may induce mitochondrial damage in neurons in vitro (Nolan and Mallal 2004). In conclusion, neurotoxicity as a result of prolonged exposure to NRTIs/NtRTIs should be monitored as a possible contributing factor to neurological deficits, especially since TDF and FTC are emerging as viable pre-exposure and post-exposure prophylaxis options.

\subsection{Non-nucleoside reverse transcriptase inhibitors \\ 5.3.1 Mechanism}

Like NRTIs/NtRTIs, nNRTIs block the conversion of the viral RNA genome to a doublestranded DNA; however, the mechanism of action differs. nNRTIs bind to the allosteric, hydrophobic site of HIV RT, inhibiting its catalytic activity (de Bethune 2010). In addition, nNRTIs are not excised by pyrophosphates, which is a mechanism by which NRTI resistance can develop (de Bethune 2010). Thus, nNRTIs can circumvent certain NRTI-associated resistance mechanisms employed by the virus. However, it is now apparent that viral resistance to nNRTI still occurs, and has become a concern, especially in patients who are either ARV-experienced with previous exposure to nNRTIs or in those who are considered for nNRTI monotherapy. Further, there is an increased risk of cross-resistance development to NRTIs which can be triggered by the presence of nNRTI-associated mutations (de Bethune 2010). Thus, at least two NRTIs/NtRTIs need to be administered with nNRTIs, to achieve complete viral suppression and prevent resistance development.

\subsubsection{Clinical use}

Nevirapine (NVP), efavirenz (EFV) and etravirine (ETR) are the three nNRTIs currently approved for use. Efavirenz (EFV) is the nNRTI of choice in developed countries, whereas nevirapine (NVP) is included in initial cART regimens in resource limited countries. Additionally, NVP is used in the prevention of mother to child transfer, as EFV in pregnant patients is not recommended in the first trimester, due to the increased risk of neural tube defects associated with EFV (De Santis, Carducci et al. 2002). ETR is increasingly incorporated into CART regimens in recent years for treatment-experienced patients, as it maintains viral suppression in the presence of nNRTI-related resistance mutations, and has high tolerability with fewer side effects (de Bethune 2010).

\subsubsection{Side effects}

The side effects due to NVP include mild-to-moderate rash and hypersensitivity-induced hepatotoxicity, which can resolve spontaneously even when the therapy is continued (de 
Bethune 2010). One major presentation of nNRTI related toxicity is neuropsychiatric disturbances. While NVP-induced CNS side effects, including impaired consciousness and psychotic episodes, have been rare, $40-70 \%$ patients on an EFV-containing CART regimen present with a range of CNS-related symptoms, such as insomnia, mood changes, and impaired attention span and concentration (Arendt, de Nocker et al. 2007).

\subsubsection{Considerations for the CNS}

While the exact mechanisms involved in EFV- and NVP-associated CNS side effects are not known, data from two recent studies have shown that chronic NVP or EFV administration in mice inhibited mitochondrial respiratory chain and creatine kinase activity in vivo (Streck, Scaini et al. 2008; Streck, Ferreira et al. 2011). As NVP and EFV show the highest CPE among all ARVs for which data is available (table 1), the changes in the energy metabolism these ARVs cause may affect particularly vulnerable cell populations in the brain, such as neurons with their high energy demands. Interestingly, a recent large scale study has shown that switching from an EFV containing cART to an ETR-based cART improved severe CNS side effects in ARV-experienced patients (Waters, Fisher et al. 2011). As will be mentioned in subsection 6.3, NVP and EFV are inducers of drug metabolism enzyme, CYP3A4, more so than ETR (Kakuda, Schöller-Gyüre et al. 2011), and may partially account for the partial alleviation in CNS-related side effects observed.

\subsection{Protease inhibitors}

\subsubsection{Mechanism}

HIV protease inhibitors (PIs) are peptidomimetics, and inhibit viral proteases that are needed for virus maturation and assembly. Ten protease inhibitors are currently in use: indinavir, ritonavir, saquinavir, lopinavir, atazanavir, nelfinavir, amprenavir, fosamprenavir, tipranavir and darunavir. PIs have been shown to control plasma viral replication and improve immunological parameters as effectively as nNRTIs; thus they form an essential component of cART regimens across most patient populations (Warnke, Barreto et al. 2007). Several factors are taken into consideration when choosing a specific PI to be included in a cART regimen: prior use of ARVs, adherence and cost-effectiveness, preexisting conditions, and side effects are a few among these, and will be discussed below.

\subsubsection{Clinical use}

PIs are recommended for use with the NRTI/NtRTI backbone in initial cART regimens, just like nNRTIs (MacArthur, Novak et al. 2006). The decision between an nNRTI- and a PIbased cART regimen depends on several factors, such as cardiovascular risks (insulin resistance, dyslipidemia), liver disease (hepatitis B and C included), pregnancy, neuropsychiatric disease risk/history, potential drug-drug interactions, and emergence of resistant virus. When PIs are included in the cART regimen, ritonavir is added to increase plasma concentrations of the first PI, as it is a potent inhibitor of CYP3A4, a major drug metabolizing enzyme ( $\mathrm{Xu}$ and Desai 2009); the aim is better viral control and decreased resistance development. In order to decrease the pill burden and increase adherence, new drug formulations that include two PIs are used increasingly in clinical practice. The most commonly used PI combination is lopinavir/ritonavir, which is recommended for use during pregnancy as well as in pediatric HIV cases. On the other hand, all combinations other than saquinavir/ritonavir can be used in hepatitis B-and C-infected patients (Stephan, 
Carlebach et al. 2007). It should be noted that the virological failure due to lopinavir/ritonavir containing regimens are less associated with the emergence of PIresistance (Prosperi, Zazzi et al. 2010), whereas nelfinavir has a lower barrier for PI-resistant virus emergence (Theys, Deforche et al. 2010). Lastly, regimens that include atazanavir/ritonavir and darunavir/ritonavir have less detrimental cardiovascular side effects compared with lopinavir/ritonavir-containing regimens (Hill, Sawyer et al. 2009). Thus, recently revised recommendations suggest atazanavir/ritonavir and darunavir/ritonavir as part of the initial cART regimen.

\subsubsection{Side effects}

Adverse effects associated with PIs are dyslipidemia, insulin resistance, and lipohypertrophy (Hui 2003). Numerous studies have shown that PIs alter lipid metabolism, and lead to dysregulation of blood lipid levels, which in itself increases risk of cardiovascular complications. Further, PIs induce oxidative and endoplasmic reticulum stress in macrophages, inducing these cells to release cytokines (CCL2, CCL3 (macrophage inflammatory protein-1 $\alpha$, MIP-1 $\alpha$ ), TNF- $\alpha$ and IL-1 $\beta$ ) and ROS (Touzet and Philips 2010). These effects of PIs on macrophages are readily observed as increased risk for atherosclerosis and are more commonly associated with lopinavir/ritonavir-including regimens (Hill, Sawyer et al. 2009).

\subsubsection{Considerations for the CNS}

Most PIs, including ritonavir and saquinavir, are highly lipophilic and strongly bind to plasma proteins; thus, they are predicted to have low CSF concentrations (table 1). These predictions are backed by various in vitro and in vivo studies (Lledo-Garcia, Nacher et al. 2007; Best, Letendre et al. 2009). However, a study conducted in an in situ guinea pig model suggests that ritonavir can achieve choroid plexus and parenchymal concentrations that are comparable to plasma levels, mainly by diffusion through the blood-choroid plexus barrier; interestingly CSF levels remain lower than those measured in the choroid plexus and the parenchymal compartments (Anthonypillai, Sanderson et al. 2004). Further, altered drug transport across the BBB due to viral proteins and neuroinflammation, in addition to a leaky $\mathrm{BBB}$ and blood-CSF barrier during the course of infection, can affect the actual CNS concentrations of ARVs. In the presence of a dysfunctioning BBB, PIs may induce indirect neuronal damage/death by triggering an inflammatory or oxidative stress response in macrophages in the CNS. Interestingly, one study has reported CNS-related side effects of PIs in an animal model where mice received ritonavir at doses comparable to those in cART regimens (Huisman, Smit et al. 2003). In that study, 2 out of 7 mice that received ritonavir co-administered with an inhibitor of the drug efflux protein p-gp developed the CNSspecific symptoms, ataxia and tremor, before death one hour following treatment. Thus, long-term effects of PIs on CNS-specific toxicities warrant further investigation.

\subsection{Integrase inhibitors}

\subsubsection{Mechanism}

Integrase inhibitors are a recently developed class of ARVs, which block the integrase enzyme that facilitates viral DNA integration into the host DNA. Currently, raltegravir is the only clinically approved drug of its class, and there are 3 more integrase inhibitors currently in different phases of clinical trials. 


\subsubsection{Clinical use and side effects}

The first clinical studies examining the efficacy and safety of raltegravir have shown that it facilitates viral suppression when added to the optimized regimens, even in patients with poor prognostic factors (Burger 2010). Further studies have shown that raltegravir efficacy was comparable to that of EFV when either drug is added to standard cART regimens (Powderly 2010; Steigbigel, Cooper et al. 2010). In addition, raltegravir is associated with few resistance mutations so far (Varghese, Liu et al. 2010), and its side effects are usually limited (Burger 2010). In fact, studies in rat hepatocytes in vitro and in mouse liver in vivo have suggested that the addition of raltegravir may inhibit PI-induced hepatotoxicity by blocking endoplasmic reticulum stress and lipid accumulation (Cao, $\mathrm{Hu}$ et al. 2010). Further, raltegravir does not appear to have an effect on drug metabolizing enzymes, which limits its possible drug-drug interactions. Thus, the FDA has now expanded its recommendations for raltegravir use in both ARV-naïve and ARV-experienced patients.

\subsubsection{Considerations for the CNS}

Raltegravir is a potential substrate of p-gp (Zembruski, Buchel et al. 2011), which would limit the entrance of this drug into the CNS. However, there are currently two clinical studies that have reported that raltegravir can achieve therapeutically relevant CSF concentrations (Yilmaz, Gisslen et al. 2009; Croteau, Letendre et al. 2010). Thus, raltegravir is given a CPE score of 0.5 . While future studies are needed to determine its access to the CNS over the long-term, raltegravir appears to be a safe drug to use in the prevention and treatment of HAND, considering its minimal toxic effects in the periphery and its favorable $\mathrm{CPE}$ score, and that fewer viral resistance mutations hamper its efficacy. However, its long term effects on CNS specific cell populations are not known and caution should be exercised due to its high CPE score.

\section{Determinants of CNS drug penetrance}

The anatomical barriers between the CNS and the periphery are critical for protection of the brain from a multitude of factors; however, HIV appears to be able to circumvent these barriers. Early CNS seeding with HIV continues as a major obstacle in the efforts to eliminate the viral reservoirs throughout the body with the use of cART. In addition to the considerations regarding how long to delay the start of cART following infection, tailored cART regimens with better CNS penetrance, higher CSF ARV concentrations, and better parenchymal distribution are being increasingly sought after in an attempt to prevent and manage HAND. As the methodologies to determine the actual drug concentrations in the CSF and parenchyma of patients are not feasible, CPE algorithms are increasingly considered in determining which ARV drugs to include in a regimen to achieve therapeutically relevant CNS concentrations. Currently, the CPE score of a given ARV is based on the chemical structure, pharmacodynamics, pharmacokinetics, and available clinical correlative data (Letendre, Marquie-Beck et al. 2008; Marra, Zhao et al. 2009; Smurzynski, Wu et al. 2011). In this section, we will summarize the factors that determine the CPE score of ARVs in the presence of HAND.

In general, for an ARV to have a high CPE score, it needs to be small in size (molecular mass below 400-500 kDa), with high lipid solubility and low protein binding, and it should not be a substrate for drug efflux or transport proteins, namely $\mathrm{p}$-gp and multidrug resistance protein-1 (MRP-1). The integrity of the BBB, which HIV infection can compromise, should 
also be considered in predicting CSF concentrations of ARVs. Also, due to the chronic nature of HIV infection, co-morbidities such as cancer, infections, and drug and alcohol abuse can also alter BBB integrity and should be considered as confounding factors in establishing effective and safe CPE algorithms.

\subsection{Blood-brain barrier}

The brain microvascular endothelial cells (BMVEC) within the brain parenchyma are the main constituents of the BBB. The BMVECs form an elaborate tight junction network, which, acts with the underlying basal lamina, pericytes, astrocyte foot processes, and microglia, to compose the BBB and to contribute to its function. A multitude of transporters and membrane proteins expressed by BMVECs regulate the cellular and molecular exchange between the CNS and the periphery, including the delivery of pharmaceuticals across the BBB into the brain parenchyma (Wolburg, Noell et al. 2009).

The accumulation of serum proteins in the CSF and brain parenchyma of HAND patients indicates that BBB dysfunction occurs in these individuals (Roberts, Buckner et al. 2010). Both in vitro and in vivo studies have shown that the viral proteins, gp120 and Tat, can impair BBB integrity by induction of oxidative stress in BMVECs (Louboutin, Agrawal et al. 2010). In addition, the disruption of tight junctions, loss of membrane glycoproteins, and apoptosis of BMVECs, as well as pericytic and perivascular astrocytic injury, have been reported following HIV infection (Persidsky, Stins et al. 1997). Given that predictions indicate that HIV-infected monocyte transmigration across the BBB will occur multiple times during the course of infection even with the successful control of peripheral VL (HIV RNA $<50$ copies $/ \mathrm{ml}$ ), the continued assault on the BBB integrity in the presence of neuroinflammation is expected to exacerbate $\mathrm{BBB}$ dysfunction. Other factors that can increase the permeability of BBB are concomitant use of other pharmaceuticals for coexisting infections, substance and alcohol abuse, and co-infections.

The basal ganglia, hippocampus, and frontal cortex are the regions that show the most striking evidence of neuroinflammation and neuronal damage in the HIV-infected brain. This regional preference of HAND correlates with the proximity of these structures to the $\mathrm{BBB}$, the dysfunction of which has multiple implications in HAND pathogenesis, clinical course, and response to treatment. First, the breakdown of this natural barrier is likely to increase the recruitment of HIV-infected monocytes from the periphery, hampering the efforts to limit neuroinflammation. Further, the risk for secondary infections increases with compromised BBB integrity. Further, altered functions of drug efflux and transport proteins are likely to affect ARV penetrance into the CNS, and consequently increase the possibility of ARV-associated toxicities in this compartment. Thus, in situations where exacerbated BBB compromise is likely, such as substance use, cART regimens with the least CNS-specific toxicities should be considered.

\subsection{Drug transport across BBB}

Complex drug efflux and transport mechanisms, which are controlled by a number of proteins that are expressed mainly on the BMVEC membrane, ultimately determine the effectiveness of any given ARV in gaining access to the CNS. P-gp and MRP-1 are the two major and well-characterized members of this regulatory mechanism. P-gp is a membrane bound, ATP-dependant drug efflux pump and regulates concentration-time profiles of its substrates, which range from amphipathic lipid-soluble compounds and metabolites to 
certain classes of ARVs, including most PIs and raltegravir (Urquhart and Kim 2009). PIs are both substrates and inhibitors of $\mathrm{p}$-gp, while raltegravir appears to be a substrate but not an inhibitor of p-gp (Huisman, Smit et al. 2000). Ritonavir and saquinavir are the most extensively evaluated PIs in this regard. Findings from in vitro and in vivo studies show that p-gp limits the permeability of both ritonavir and saquinavir across the BBB. Interestingly, these drugs increase the expression of p-gp as well (Huisman, Smit et al. 2000). Ritonavir is added to most cART regimens in both ARV-naïve and ARV-experienced patients in order to exploit its ability to boost plasma concentrations of other ARVs (Xu and Desai 2009). However, the ability of ritonavir to increase the expression of p-gp can limit CNS access of concomitantly administered ARVs that are also p-gp substrates. Further, HIV infection can increase p-gp expression in T cells and monocytic cell lines and this capacity may extend to other cell-types, further contributing to the limited penetrance of ARVs to the CNS (Zhong, Hennig et al. 2010).

MRP-1 provides an additional level of regulation of drug concentrations in the CNS. This BBB-resident protein mainly transports neutral drugs conjugated to glutathione, sulfate, or glucuronate, in addition to negatively charged anionic drugs and organic anionic drugs (Dallas, Miller et al. 2006). Numerous in vitro and in vivo studies show that PIs, as well as a number of NRTIs and nNRTIs, can inhibit MRP-1 function. A recent study reports that two NRTIs included in the recommended cART regimens, TDF and FTC, as well as the two most commonly used nNRTIs, EFV and NVP, can inhibit MRP-1 in a concentration-dependent manner in vitro (Weiss, Theile et al. 2007). Further, while TDF concentration in the CSF is limited, as measured in patient samples (Anthonypillai, Gibbs et al. 2006), clinical data show that TDF or FTC-including regimens are associated with better neurological outcomes (Winston, Duncombe et al. 2010), suggesting that the effect of cART on MRP-1 can influence the outcomes of antiretroviral therapy in the CNS.

\subsection{Pharmacokinetics}

In the liver, where the majority of drug metabolism occurs via the Cytochrome P450 (CYP) family of enzymes, Cytochrome P450 3A4 (CYP3A4) is the major enzyme that metabolizes the vast majority of drugs, including ARVs (Lakhman, Ma et al. 2009). Conversely, some drugs, including ARVs can enhance or inhibit the activity of CYP3A4 and can precipitate decreased drug efficacy or increased drug concentrations and toxicity, respectively, when added to the existing treatment plan (Fichtenbaum and Gerber 2002). For example, several non-ARV drugs prescribed concurrently with ARVs for co-existing conditions, such as macrolide antibiotics, cholesterol-lowering drugs, and calcium channel blockers, are CYP3A4 inhibitors, as is ritonavir. The addition of one of the aforementioned non-ARV drugs to a cART regimen that already includes ritonavir necessitates the lowering of ARV doses. EFV and NVP, on the other hand are strong inducers of CYP3A4, further complicating treatment strategies (Mugundu, Hariparsad et al. 2010). Data from a guinea pig model of drug diffusion showed that NVP limited CSF distribution of ritonavir (Anthonypillai, Sanderson et al. 2004). This example further highlights the importance of drug-drug interactions in determining the CPE score of a cART regimen.

Intriguingly, CYP2B6, another member of the CYP family, is also induced by EFV and NVP (Ngaimisi, Mugusi et al. 2010). CYP2B6 is expressed in a number of extrahepatic tissues in addition to the liver, including the neurons and astrocytes of the different brain regions (Wang and Tompkins 2008). Thus, the predicted concentrations of drugs that are CYP2B6 substrates in the CNS can be compounded by the concurrent use of EFV or NVP. On the 
other hand, the activity of CYP2B6 varies widely among individuals due to the common occurrence of SNPs (Elens, Vandercam et al. 2010), as well as its transcriptional regulation (Wang and Tompkins 2008), and pharmacogenomic profiling may be necessary for successful dosing for regimens that include EFV and NVP.

In summary, the factors discussed in this section contribute to the CPE scoring and provide a starting point for clinicians for devising the most effective cART regimen to control HAND. However, dose adjustments and drug replacements have become a necessary part of the treatment plans while trying to find a balance between maximum efficiency and minimum side effects. At the present, the implications of these changes during the long course of HIV infection are not known; however, several clinical studies suggest that insufficient suppression of CSF VL, which can arise from fluctuations in the CSF ARV concentrations, is associated with treatment failure, i.e. emergence of resistant virus, worsening of neurological symptoms. Thus, utmost effort should be made to minimize such fluctuations in order to decrease the prevalence of HAND.

\section{The impact of CPE scores on HAND}

To date, six studies have investigated the effectiveness of CPE scoring in the prevention and management of HAND (Letendre, Marquie-Beck et al. 2008; Cysique, Vaida et al. 2009; Marra, Zhao et al. 2009; Garvey, Winston et al. 2011; Lanoy, Guiguet et al. 2011; Smurzynski, Wu et al. 2011). The most important conclusion these studies suggest is that the use of ARVs with high CPE scores is beneficial for suppressing CSF HIV RNA levels and for improving neurocognitive deficits. Further, this correlation between CPE scores and neurological improvements is independent of the success of the given regimen at suppressing peripheral VL. Interestingly, data suggest that ARV-naive patients show better improvement in neurocognitive deficits than ARV-experienced patients, likely due to the existence of drugresistant virus harbored by the latter group.

While the majority of these studies have found a positive correlation between CPE scores and neurological outcomes, a study by Marra et al. reported that CART regimens with higher CPE scores were associated with worse neurocognitive performance (Marra, Zhao et al. 2009). The authors argued that this result was most likely due to the small size of the cohort and a possible bias towards prescription of more CPE penetrant regimens to patients with worse baseline neurocognitive impairment. However, cART regimens with higher CPE scores carry a risk of neurotoxicity in the already compromised brains of patients.

There are surprisingly few animal studies that investigated CNS-specific side effects of ARVs; however, findings suggest that NVP and EFV can induce cognitive deficits and anxiety when administered at doses that are reflective of doses used in humans over a short term (Streck, Scaini et al. 2008; Streck, Ferreira et al. 2011). The underlying mechanisms of NVP or EFV-induced CNS side effects are not known but given that these ARVs can achieve therapeutically relevant concentrations in the CNS, the long-term exposure to these ARVs may contribute to the persistence of HAND despite successful control of VL and further investigation is warranted.

Interestingly, one study has reported that ritonavir can induce acute signs of CNS-related toxicity, including ataxia and tremor in mice followed by death within an hour, when it is administered in the presence of a p-gp inhibitor (Huisman, Smit et al. 2003). These data suggests that acute exposure to high doses of ritonavir might be neurotoxic. In addition, mice that were administered lopinavir/ritonavir for 3 weeks at clinically relevant doses showed 
significant cognitive impairment, as determined by a multi-unit T-maze (Pistell, Gupta et al. 2010). Further, PIs cause increases in inflammatory markers via oxidative and endoplasmic reticulum stress response of macrophages (Touzet and Philips 2010). Thus, some of the CNSrelated toxicities observed in animal models might be a result of activated macrophages by these ARVs. In the presence of a neuroinflammatory environment, the recruitment ARVactivated macrophages from the periphery in the presence of a compromised BBB can potentiate indirect neurotoxicity due to these ARVs. In addition, as mentioned in section 5.4, ritonavir concentrations in the brain parenchyma might be higher than predicted by the classical determinants of CPE (Anthonypillai, Sanderson et al. 2004), and direct neurotoxicity via oxidative stress can further precipitate neuronal damage and death.

In summary, in a setting where long-term administration of cART is inevitable to control infection and as a prophylactic measure, animal studies which better represent the inflammatory conditions observed in HIV-infected brain are needed to more successfully predict the CPE scores of ARVs and determine the most efficient combinations with minimal CNS related toxicities. Further, large-scale, longitudinal studies will be instrumental to tease out the contribution of ARV-related neurotoxicities to the persistence of HAND.

\section{CNS-specific co-morbidities and cART effectiveness}

Since its inception, cART has changed the landscape of HIV infection. However, it has also introduced unique challenges in the management of HIV. Due to longer life expectancy, comorbidities such as substance abuse, chronic co-infections, and aging have become major contributors to HAND development and persistence. These co-morbidities need to be taken into consideration when cART regimens are determined on a patient-by-patient base.

\subsection{Drugs of abuse}

Perhaps the most important HIV associated co-morbidity is illicit drug use. For example, HIV-infected patients using methamphetamine (METH) show more neurocognitive deficits, compared to those not using METH (Nath 2010). These findings are not surprising, as illicit drugs, such as morphine and methamphetamine, alter the BBB permeability through direct damage to the BMVECs (Ramirez, Potula et al. 2009). In addition, these drugs exert neurotoxic effects in the CNS through oxidative stress and mitochondrial dysfunction (Yamamoto, Moszczynska et al. 2010). As mentioned in previous sections, BBB dysfunction and neurotoxicity are observed during the course of HIV infection as well as in response to ARV exposure and include similar mechanisms. Considering that the prevalence of HIV infection is $12-17 \%$ among illicit drug users (Cadet and Krasnova 2007), animal and clinical studies are essential to tailor cART regimens that reach sufficient CSF concentrations and yet do not augment neurotoxicity that might already be occuring in response to drug abuse.

\subsection{Chronic alcohol use}

A second co-morbidity that needs to be considered in the era of CART is chronic alcohol use. Chronic alcohol consumption has been established as a major confounding factor in the development of HAND (Durazzo, Rothlind et al. 2007; Miguez-Burbano, Lewis et al. 2008; Miguez-Burbano, Nair et al. 2009). Extensive studies have shown that chronic ethanol exposure causes neuronal damage and death (Brust 2010). Further, ethanol directly damages 
the BBB structure, and elicits a pro-inflammatory response in the parenchyma, as detected by increases in CCL2 and IL1- $\beta$, and increased ROS accumulation in the hippocampus(Shiu, Barbier et al. 2007; Qin, He et al. 2008). These effects are expected to be augmented in the presence of HIV infection. Further, ARV-induced neurotoxicity due to loss of BBB integrity in the setting of neuroinflammation is a possibility over the long term. Finally, chronic alcohol use will impair adherence to cART regimens. Thus, chronic alcohol use remains an important obstacle in the eradication of HAND.

\subsection{Co-infections}

The possible CNS-related effects of co-infections also need to be considered in HIV-infected population. Hepatitis $\mathrm{B}$ and $\mathrm{C}(\mathrm{HBV}$ and $\mathrm{HCV})$ were major co-morbidity and mortality factors in the pre-cART era. cART not only improved immunological functions, altering the detrimental effects of these viruses on the immune system, but also helped control the hepatitis virus replication to a certain extent. However, mortality due to liver disease remains the second most common following AIDS-related deaths. Recent data from AntiHIV study group (D:A:D) revealed that, of all the deaths that are due to liver disease, $66 \%$ were a result of $\mathrm{HCV}$, while $17 \%$ was due to $\mathrm{HBV}$, and $3 \%$ was related to cART-related hepatotoxicity (Turner, Bansi et al. 2010). While these viruses do not target the cells of the CNS and there is no direct evidence of hepatitis as a contributing factor for the persistence of HAND; there is evidence that viral suppression and CD4 cell recovery is not as successful in the presence of $\mathrm{HCV}$ or HBV. These findings might partially explain the sporadic data suggesting that worse neurocognitive impairment is observed in HIV-HCV co-infected patients (Winston, Duncombe et al. 2010).

\subsection{Aging}

The contribution of cART to the increased life span of HIV-infected individuals cannot be overstated. However, longer life expectancy has brought new complications to the management of disease, one of which is the age-related metabolic and functional changes in the CNS. The neuroinflammation is still observed in the hippocampus of patients with suppressed CNS and peripheral VL, in addition to the accumulation of phosphorylated Tau, and intracellular and extracellular $\beta$-amyloid $(\mathrm{A} \beta)$ in the frontal cortex and the hippocampus(Brew, Pemberton et al. 2005; Green, Masliah et al. 2005; Anthony, Ramage et al. 2006; Achim, Adame et al. 2009). Interestingly, while increased accumulation of amyloid precursor protein (APP) was observed in the brains of HIV-infected individuals, the presence of phospho-Tau and $\mathrm{A} \beta$ were not demonstrated before the implementation of cART. Among the factors that may contribute to this shift are increased life span and prolonged neuroinflammation. It is established that risk of developing Alzheimer Disease (AD) increases with age. In addition, prolonged and/or uncontrolled neuroinflammation due to the emergence of new co-morbidities, adjustments in the cART regimens, and direct and indirect cART-related neurotoxicity can further exacerbate the neuropathological changes that classically occur in the aging brain.

Both HAND and AD brains show increases in markers of neuroinflammation, oxidative stress, and endoplasmic reticulum stress (Lindl, Akay et al. 2007). Interestingly, several in vitro and in vivo studies have shown that ARVs can lead to cellular toxicity via oxidative stress and endoplasmic reticulum stress (Zhou, Gurley et al. 2006; Gavilan, Pintado et al. 2009; Salminen, Kauppinen et al. 2009). Interestingly, these aforementioned stress pathways 
have been shown to increase $A \beta$ secretion both in vitro and in vivo (Velliquette, O'Connor et al. 2005; O'Connor, Sadleir et al. 2008). Thus, age-related changes might be partially due to cART-related amyloidogenic processes, and the possible contributions of cART to these agerelated changes need to be studied while establishing regimens with high CNS concentrations.

\section{Adjunctive therapies}

In addition to the utilization of the CPE scoring system, alternative delivery methods such as nanoparticle delivery are being explored. Further, adjunctive therapies are currently being studied for their efficiency in alleviating neuroinflammation, and decreasing neuronal damage and death. While the initial data obtained from these studies suggest limited success, the results are encouraging and constitute a newly expending field in the management of HAND.

\subsection{Anti-excitotoxicity agents}

The excitotoxins, such as glutamate and quinolinic acid that accumulate as a result of activated and/or infected macrophages and astrocytes cause direct neuronal dysfunction via activation of the NMDA receptor, as mentioned previously(O'Donnell, Agrawal et al. 2006; Lindl, Marks et al. 2010). Thus, memantine, an NMDA receptor antagonist that is approved for use in AD (Lipton and Chen 2004) is explored for its neuroprotective effects in HAND (Schifitto, Navia et al. 2007). Clinical trials so far report that memantine appears to be associated with few side effects, with favorable tolerance. Further, patients that received memantine showed improved neurocognitive functions. Given that memantine has shown benefits in patients with $\mathrm{AD}$, it emerges as an attractive treatment option in a patient population that increasingly consists of aging patients.

\subsection{Anti-oxidants}

Oxidative stress, as mentioned before, is a major component of HIV-induced neuroinflammation. The increases in 4-HNE, protein carbonyls, long-chain sphingomyelins and ceramides are still observed in the presence of cART (Haughey, Cutler et al. 2004; Sacktor, Haughey et al. 2004). Use of illicit drugs and ethanol can induce oxidative stress, exacerbating the accumulation of oxidative products, and ARVs might contribute to this process. Thus, antioxidants were among the first to be tested as adjuncts to ARVs. Selegiline is one such antioxidant that was tested in several small clinical trials (Sacktor, Schifitto et al. 2000; Schifitto, Zhang et al. 2007). While initial studies conducted in patients receiving selegiline and NRTIs showed slight improvement in cognitive functions, a more comprehensive study that enrolled patients who received cART reported that neither the CSF and neuroimaging markers of oxidative stress nor cognitive and functional deficits improved with selegiline over a 24 week period (Schifitto, Yiannoutsos et al. 2009). One caveat of this study is its small number of patients, while a second caveat is the lack of stratification of patients according to the CPE scores. Thus, future studies that will take CPE scores of the CART regimens into account are necessary to better assess the efficacy of selegiline. This approach of utilizing antioxidants will be beneficial at multiple levels, such as virus-related direct and indirect neurotoxicity, ARV-related oxidative changes in the CNS, and alcohol and illicit drug-induced oxidative responses. 


\subsection{Anti-inflammatory agents}

The neuroinflammation in the brains of HIV-infected patients are partially due to the activation of macrophages in response to HIV, or co-morbid conditions such as chronic alcohol and illicit drug use. In an attempt to resolve the persistent neuroinflammation, adjunctive agents to alleviate the inflammatory component of HAND are currently sought. One such agent is minocycline, a tetracycline antibiotic with both neuroprotective and antiinflammatory effects. Its efficacy has been shown in models of traumatic and ischemic brain injury, and other neurodegenerative diseases such as $\mathrm{AD}$ and multiple sclerosis (Chen, Ona et al. 2000; Du, Ma et al. 2001; Van Den Bosch, Tilkin et al. 2002; Metz, Zhang et al. 2004). Further, it has been shown to be effective in limiting HIV replication in vitro and its close relative simian immunodeficiency virus (SIV) in vivo (Zink, Uhrlaub et al. 2005). Further, neuroinflammation markers in the CNS in the animal model of SIV encephalitis were decreased with minocycline administration. Currently, clinical trials are being conducted to assess its potential. In summary, its favorable safety profile, ease of administration and efficient BBB permeability makes it a viable adjunctive agent.

\section{Conclusion}

One of the paradoxical outcomes of cART is the persistence of HAND despite successful CNS viral control. Moreover, there seems to be a shift from overt dementia to more subtle presentations of neurocognitive impairment. Probable cART-related neuronal perturbations might contribute, and even precipitate, some of these changes in the chronic course of HAND, considering that cART regimens with higher CPE scores are prescribed in cases of higher or uncontrollable viral burden. However, the data regarding the impact of comorbidities and possible cART-related side effects on CPE scores and the success of cART on HAND need to be expanded, given the ever-expending repertoire of ARVs and the patient-tailored, dynamic cART regimens in a multiple-system therapy management to ensure more successful outcomes of cART. The information gained from such studies will help physicians to determine the most efficient cART regimens with minimal CNS side effects, and further help design and determine adjunctive therapeuticals in the management of CNS perturbations in HIV-infected patients on long-term cART.

\section{References}

(1996). "Clinical confirmation of the American Academy of Neurology algorithm for HIV-1associated cognitive/motor disorder. The Dana Consortium on Therapy for HIV Dementia and Related Cognitive Disorders." Neurology 47(5): 1247-1253.

Achim, C. L., A. Adame, et al. (2009). "Increased accumulation of intraneuronal amyloid beta in HIV-infected patients." J Neuroimmune Pharmacol 4(2): 190-199.

Achim, C. L., E. Masliah, et al. (1996). "Macrophage Activation Factors<br / >in the Brains of AIDS Patients." J NeuroAIDS 1(2): 1-16.

Achim, C. L. and C. A. Wiley (1996). "Inflammation in AIDS and the role of the macrophage in brain pathology." Current Opinion in Neurology 9(3): 221-225.

Allavena, C., G. Le Moal, et al. (2006). "Neuropsychiatric adverse events after switching from an antiretroviral regimen containing efavirenz without tenofovir to an efavirenz regimen containing tenofovir: a report of nine cases." Antivir Ther 11(2): 263-265. 
Anderson, P. L. and J. E. Rower (2010). "Zidovudine and Lamivudine for HIV Infection." Clin Med Rev Ther 2: a2004.

Anthony, I. C. and J. E. Bell (2008). "The Neuropathology of HIV/AIDS." Int Rev Psychiatry 20(1): 15-24.

Anthony, I. C., S. N. Ramage, et al. (2006). "Accelerated Tau deposition in the brains of individuals infected with human immunodeficiency virus-1 before and after the advent of highly active anti-retroviral therapy." Acta Neuropathol 111(6): 529-538.

Anthonypillai, C., J. Gibbs, et al. (2006). "The distribution of the anti-HIV drug, tenofovir (PMPA), into the brain, CSF and choroid plexuses." Cerebrospinal Fluid Research 3(1): 1 .

Anthonypillai, C., R. N. Sanderson, et al. (2004). "The Distribution of the HIV Protease Inhibitor, Ritonavir, to the Brain, Cerebrospinal Fluid, and Choroid Plexuses of the Guinea Pig." Journal of Pharmacology and Experimental Therapeutics 308(3): 912920.

Antinori, A., G. Arendt, et al. (2007). "Updated research nosology for HIV-associated neurocognitive disorders." Neurology 69(18): 1789-1799.

Arendt, G., D. de Nocker, et al. (2007). "Neuropsychiatric side effects of efavirenz therapy." Expert Opin Drug Saf 6(2): 147-154.

Bazzoli, C., V. Jullien, et al. (2010). "Intracellular Pharmacokinetics of Antiretroviral Drugs in HIV-Infected Patients, and their Correlation with Drug Action." Clinical Pharmacokinetics 49(1): 17-45 10.2165/11318110-000000000-000000000.

Best, B. M., S. L. Letendre, et al. (2009). "Low atazanavir concentrations in cerebrospinal fluid." Aids 23(1): 83-87.

Brew, B. J., L. Pemberton, et al. (2005). "CSF amyloid beta42 and tau levels correlate with AIDS dementia complex." Neurology 65(9): 1490-1492.

Brew, B. J., L. Pemberton, et al. (1997). "Levels of human immunodeficiency virus type 1 RNA in cerebrospinal fluid correlate with AIDS dementia stage." J Infect Dis 175(4): 963-966.

Brust, J. C. (2010). "Ethanol and cognition: indirect effects, neurotoxicity and neuroprotection: a review." Int J Environ Res Public Health 7(4): 1540-1557.

Burger, D. M. (2010). "Raltegravir: a review of its pharmacokinetics, pharmacology and clinical studies." Expert Opin Drug Metab Toxicol 6(9): 1151-1160.

Cadet, J. and I. Krasnova (2007). "Interactions of HIV and methamphetamine: Cellular and molecular mechanisms of toxicity potentiation." Neurotoxicity Research 12(3): 181-204.

Cao, R., Y. Hu, et al. (2010). "Prevention of HIV protease inhibitor-induced dysregulation of hepatic lipid metabolism by raltegravir via endoplasmic reticulum stress signaling pathways." J Pharmacol Exp Ther 334(2): 530-539.

Chen, M., V. O. Ona, et al. (2000). "Minocycline inhibits caspase- 1 and caspase-3 expression and delays mortality in a transgenic mouse model of Huntington disease." Nat Med 6(7): 797-801.

Cole, M. A., J. B. Margolick, et al. (2007). "Longitudinally preserved psychomotor performance in long-term asymptomatic HIV-infected individuals." Neurology 69(24): 2213-2220. 
Conant, K., A. Garzino-Demo, et al. (1998). "Induction of monocyte chemoattractant protein1 in HIV-1 Tat-stimulated astrocytes and elevation in AIDS dementia." Proc Natl Acad Sci U S A 95(6): 3117-3121.

Croteau, D., S. Letendre, et al. (2010). "Total raltegravir concentrations in cerebrospinal fluid exceed the 50-percent inhibitory concentration for wild-type HIV-1." Antimicrob Agents Chemother 54(12): 5156-5160.

Cysique, L. A., F. Vaida, et al. (2009). "Dynamics of cognitive change in impaired HIVpositive patients initiating antiretroviral therapy." Neurology 73(5): 342-348.

Dagan, T., C. Sable, et al. (2002). "Mitochondrial dysfunction and antiretroviral nucleoside analog toxicities: what is the evidence?" Mitochondrion 1(5): 397-412.

Dalakas, M. C. (2001). "Peripheral neuropathy and antiretroviral drugs." J Peripher Nerv Syst 6(1): 14-20.

Dallas, S., D. S. Miller, et al. (2006). "Multidrug resistance-associated proteins: expression and function in the central nervous system." Pharmacol Rev 58(2): 140-161.

Danial, N. N. and S. J. Korsmeyer (2004). "Cell death: critical control points." Cell 116(2): 205-219.

de Bethune, M. P. (2010). "Non-nucleoside reverse transcriptase inhibitors (NNRTIs), their discovery, development, and use in the treatment of HIV-1 infection: a review of the last 20 years (1989-2009)." Antiviral Res 85(1): 75-90.

De Santis, M., B. Carducci, et al. (2002). "Periconceptional exposure to efavirenz and neural tube defects." Arch Intern Med 162(3): 355.

Du, Y., Z. Ma, et al. (2001). "Minocycline prevents nigrostriatal dopaminergic neurodegeneration in the MPTP model of Parkinson's disease." Proc Natl Acad Sci U S A 98(25): 14669-14674.

Duarte-Rojo, A. and E. J. Heathcote (2010). "Efficacy and safety of tenofovir disoproxil fumarate in patients with chronic hepatitis B." Therap Adv Gastroenterol 3(2): 107-119.

Durazzo, T. C., J. C. Rothlind, et al. (2007). "Chronic cigarette smoking and heavy drinking in human immunodeficiency virus: consequences for neurocognition and brain morphology." Alcohol 41(7): 489-501.

Edén, A., D. Fuchs, et al. (2010). "HIV-1 Viral Escape in Cerebrospinal Fluid of Subjects on Suppressive Antiretroviral Treatment." Journal of Infectious Diseases 202(12): 1819-1825.

Elens, L., B. Vandercam, et al. (2010). "Influence of host genetic factors on efavirenz plasma and intracellular pharmacokinetics in HIV-1-infected patients." Pharmacogenomics 11(9): 1223-1234.

Ellis, R. (2010). "HIV and antiretroviral therapy: impact on the central nervous system." Prog Neurobiol 91(2): 185-187.

Ellis, R. J., R. Deutsch, et al. (1997). "Neurocognitive impairment is an independent risk factor for death in HIV infection. San Diego HIV Neurobehavioral Research Center Group." Arch Neurol 54(4): 416-424.

Everall, I., F. Vaida, et al. (2009). "Cliniconeuropathologic correlates of human immunodeficiency virus in the era of antiretroviral therapy." J Neurovirol 15(5-6): 360-370. 
Fichtenbaum, C. J. and J. G. Gerber (2002). "Interactions between antiretroviral drugs and drugs used for the therapy of the metabolic complications encountered during HIV infection." Clin Pharmacokinet 41(14): 1195-1211.

Garvey, L., A. Winston, et al. (2011). "Antiretroviral therapy CNS penetration and HIV-1associated CNS disease." Neurology 76(8): 693-700.

Gavilan, M. P., C. Pintado, et al. (2009). "Dysfunction of the unfolded protein response increases neurodegeneration in aged rat hippocampus following proteasome inhibition." Aging Cell 8(6): 654-665.

Gendelman, H. E., S. A. Lipton, et al. (1994). "The neuropathogenesis of HIV-1 infection.[see comment]. [Review] [107 refs]."

Gisolf, E. H., R. M. van Praag, et al. (2000). "Increasing cerebrospinal fluid chemokine concentrations despite undetectable cerebrospinal fluid HIV RNA in HIV-1infected patients receiving antiretroviral therapy." J Acquir Immune Defic Syndr 25(5): 426-433.

Gisolf, E. H., R. M. E. van Praag, et al. (2000). "Increasing Cerebrospinal Fluid Chemokine Concentrations Despite Undetectable Cerebrospinal Fluid HIV RNA in HIV-1Infected Patients Receiving Antiretroviral Therapy." JAIDS Journal of Acquired Immune Deficiency Syndromes 25(5): 426-433.

Giulian, D., J. Yu, et al. (1996). "Study of receptor-mediated neurotoxins released by HIV-1infected mononuclear phagocytes found in human brain." J Neurosci 16(10): 31393153.

Gonzalez-Scarano, F. and J. Martin-Garcia (2005). "The neuropathogenesis of AIDS." Nat Rev Immunol 5(1): 69-81.

Gonzalez-Scarano, F. and J. Martin-Garcia (2005). "The neuropathogenesis of AIDS. [Review] [173 refs]."

Gorry, P. R., C. Ong, et al. (2003). "Astrocyte infection by HIV-1: mechanisms of restricted virus replication, and role in the pathogenesis of HIV-1-associated dementia." Curr HIV Res 1(4): 463-473.

Grant, R. M. (2010). "Antiretroviral agents used by HIV-uninfected persons for prevention: pre- and postexposure prophylaxis." Clin Infect Dis 50 Suppl 3: S96-101.

Green, D. A., E. Masliah, et al. (2005). "Brain deposition of beta-amyloid is a common pathologic feature in HIV positive patients." Aids 19(4): 407-411.

Hagberg, L., P. Cinque, et al. (2010). "Cerebrospinal fluid neopterin: an informative biomarker of central nervous system immune activation in HIV-1 infection." AIDS Research and Therapy 7(1): 15.

Haughey, N. J., R. G. Cutler, et al. (2004). "Perturbation of sphingolipid metabolism and ceramide production in HIV-dementia." Ann Neurol 55(2): 257-267.

Heaton, R. K., D. B. Clifford, et al. "HIV-associated neurocognitive disorders persist in the era of potent antiretroviral therapy: CHARTER Study." Neurology 75(23): 2087-2096.

Heaton, R. K., D. B. Clifford, et al. (2010). "HIV-associated neurocognitive disorders persist in the era of potent antiretroviral therapy: CHARTER Study." Neurology 75(23): 2087-2096.

Hill, A. and W. Sawyer (2009). "Effects of nucleoside reverse transcriptase inhibitor backbone on the efficacy of first-line boosted highly active antiretroviral therapy 
based on protease inhibitors: meta-regression analysis of 12 clinical trials in 5168 patients." HIV Med 10(9): 527-535.

Hill, A., W. Sawyer, et al. (2009). "Effects of first-line use of nucleoside analogues, efavirenz, and ritonavir-boosted protease inhibitors on lipid levels." HIV Clin Trials 10(1): $1-12$.

Hu, S., W. S. Sheng, et al. (2009). "Preferential sensitivity of human dopaminergic neurons to gp120-induced oxidative damage." J Neurovirol 15(5-6): 401-410.

Hui, D. Y. (2003). "Effects of HIV protease inhibitor therapy on lipid metabolism." Progress in Lipid Research 42(2): 81-92.

Huisman, M. T., J. W. Smit, et al. (2000). "Significance of P-glycoprotein for the pharmacology and clinical use of HIV protease inhibitors." Aids 14(3): 237-242.

Huisman, M. T., J. W. Smit, et al. (2003). "Assessing safety and efficacy of directed Pglycoprotein inhibition to improve the pharmacokinetic properties of saquinavir coadministered with ritonavir." J Pharmacol Exp Ther 304(2): 596-602.

Joly, V., K. Jidar, et al. (2010). "Enfuvirtide: from basic investigations to current clinical use." Expert Opin Pharmacother 11(16): 2701-2713.

Kakuda, T. N., M. Schöller-Gyüre, et al. (2011). "Pharmacokinetic Interactions between Etravirine and Non-Antiretroviral Drugs." Clinical Pharmacokinetics 50(1): 25-39 10.2165/11534740-000000000-000000000.

Kaul, M., J. Zheng, et al. (2005). "HIV-1 infection and AIDS: consequences for the central nervous system. [Review] [199 refs]."

Kohler, J. J. and W. Lewis (2007). "A brief overview of mechanisms of mitochondrial toxicity from NRTIs." Environmental and Molecular Mutagenesis 48(3-4): 166-172.

Kolson, D. L. (2002). "Neuropathogenesis of central nervous system HIV-1 infection." Clin Lab Med 22(3): 703-717.

Kromdijk, W., A. D. Huitema, et al. (2010). "Treatment of HIV infection with the CCR5 antagonist maraviroc." Expert Opin Pharmacother 11(7): 1215-1223.

Kusdra, L., D. McGuire, et al. (2002). "Changes in monocyte/macrophage neurotoxicity in the era of HAART: implications for HIV-associated dementia." Aids 16(1): 31-38.

Lakhman, S. S., Q. Ma, et al. (2009). "Pharmacogenomics of CYP3A: considerations for HIV treatment." Pharmacogenomics 10(8): 1323-1339.

Lanoy, E., M. Guiguet, et al. (2011). "Survival after neuroAIDS." Neurology 76(7): 644-651.

Letendre, S., J. Marquie-Beck, et al. (2008). "Validation of the CNS Penetration-Effectiveness rank for quantifying antiretroviral penetration into the central nervous system." Arch Neurol 65(1): 65-70.

Letendre, S. L., R. J. Ellis, et al. (2010). "Neurologic complications of HIV disease and their treatment." Top HIV Med 18(2): 45-55.

Lieberman-Blum, S. S., H. B. Fung, et al. (2008). "Maraviroc: a CCR5-receptor antagonist for the treatment of HIV-1 infection." Clin Ther 30(7): 1228-1250.

Lindl, K. A., C. Akay, et al. (2007). "Expression of the endoplasmic reticulum stress response marker, BiP, in the central nervous system of HIV-positive individuals." Neuropathol Appl Neurobiol 33(6): 658-669.

Lindl, K. A., D. R. Marks, et al. (2010). "HIV-associated neurocognitive disorder: pathogenesis and therapeutic opportunities." J Neuroimmune Pharmacol 5(3): 294-309. 
Lipton, S. A. and H. S. Chen (2004). "Paradigm shift in neuroprotective drug development: clinically tolerated NMDA receptor inhibition by memantine." Cell Death Differ 11(1): 18-20.

Liu, N. Q., A. S. Lossinsky, et al. (2002). "Human immunodeficiency virus type 1 enters brain microvascular endothelia by macropinocytosis dependent on lipid rafts and the mitogen-activated protein kinase signaling pathway." J Virol 76(13): 6689-6700.

Lledo-Garcia, R., A. Nacher, et al. (2007). "Bioavailability and pharmacokinetic model for ritonavir in the rat." J Pharm Sci 96(3): 633-643.

Louboutin, J. P., L. Agrawal, et al. (2010). "HIV-1 gp120-induced injury to the blood-brain barrier: role of metalloproteinases 2 and 9 and relationship to oxidative stress." J Neuropathol Exp Neurol 69(8): 801-816.

MacArthur, R. D., R. M. Novak, et al. (2006). "A comparison of three highly active antiretroviral treatment strategies consisting of non-nucleoside reverse transcriptase inhibitors, protease inhibitors, or both in the presence of nucleoside reverse transcriptase inhibitors as initial therapy (CPCRA 058 FIRST Study): a longterm randomised trial." Lancet 368(9553): 2125-2135.

Marra, C. M., Y. Zhao, et al. (2009). "Impact of combination antiretroviral therapy on cerebrospinal fluid HIV RNA and neurocognitive performance." Aids 23(11): 13591366.

Masliah, E., R. K. Heaton, et al. (1997). "Dendritic injury is a pathological substrate for human immunodeficiency virus-related cognitive disorders. HNRC Group. The HIV Neurobehavioral Research Center." Ann Neurol 42(6): 963-972.

McArthur, J. C. (2004). "HIV dementia: an evolving disease." J Neuroimmunol 157(1-2): 3-10.

McArthur, J. C., M. P. McDermott, et al. (2004). "Attenuated Central Nervous System Infection in Advanced HIV/AIDS With Combination Antiretroviral Therapy." Arch Neurol 61(11): 1687-1696.

Metz, L. M., Y. Zhang, et al. (2004). "Minocycline reduces gadolinium-enhancing magnetic resonance imaging lesions in multiple sclerosis." Ann Neurol 55(5): 756.

Miguez-Burbano, M. J., J. E. Lewis, et al. (2008). "Cognitive performance and the thymus among HIV-infected subjects receiving HAART." Biologics 2(2): 321-327.

Miguez-Burbano, M. J., M. Nair, et al. (2009). "The role of alcohol on platelets, thymus and cognitive performance among HIV-infected subjects: are they related?" Platelets 20(4): 260-267.

Moore, J. D., N. J. Rothwell, et al. (2002). "Involvement of caspases and calpains in cerebrocortical neuronal cell death is stimulus-dependent." Br J Pharmacol 135(4): 1069-1077.

Mugundu, G. M., N. Hariparsad, et al. (2010). "Impact of ritonavir, atazanavir and their combination on the CYP3A4 induction potential of efavirenz in primary human hepatocytes." Drug Metab Lett 4(1): 45-50.

Nath, A. (2010). "Human immunodeficiency virus-associated neurocognitive disorder: pathophysiology in relation to drug addiction." Ann N Y Acad Sci 1187: 122-128.

Navia, B. A., E. S. Cho, et al. (1986). "The AIDS dementia complex: II. Neuropathology." Annals of Neurology 19(6): 525-535.

Nelson, M. and M. Schiavone (2004). "Emtricitabine (FTC) for the treatment of HIV infection." Int J Clin Pract 58(5): 504-510. 
Neuenburg, J. K., H. R. Brodt, et al. (2002). "HIV-related neuropathology, 1985 to 1999: rising prevalence of HIV encephalopathy in the era of highly active antiretroviral therapy." J Acquir Immune Defic Syndr 31(2): 171-177.

Ngaimisi, E., S. Mugusi, et al. (2010). "Long-term efavirenz autoinduction and its effect on plasma exposure in HIV patients." Clin Pharmacol Ther 88(5): 676-684.

Nolan, D. and S. Mallal (2004). "Complications associated with NRTI therapy: update on clinical features and possible pathogenic mechanisms." Antivir Ther 9(6): 849-863.

O'Connor, T., K. R. Sadleir, et al. (2008). "Phosphorylation of the translation initiation factor eIF2alpha increases BACE1 levels and promotes amyloidogenesis." Neuron 60(6): 988-1009.

O'Donnell, L. A., A. Agrawal, et al. (2006). "Human immunodeficiency virus (HIV)-induced neurotoxicity: roles for the NMDA receptor subtypes." J Neurosci 26(3): 981-990.

O'Donnell, L. A., A. Agrawal, et al. (2006). "Human immunodeficiency virus (HIV)-induced neurotoxicity: roles for the NMDA receptor subtypes." Journal Of Neuroscience.

Owe-Larsson, B., L. Sall, et al. (2009). "HIV infection and psychiatric illness." Afr J Psychiatry (Johannesbg) 12(2): 115-128.

Patel, I. H., X. Zhang, et al. (2005). "Pharmacokinetics, pharmacodynamics and drug interaction potential of enfuvirtide." Clin Pharmacokinet 44(2): 175-186.

Persidsky, Y., M. Stins, et al. (1997). "A model for monocyte migration through the bloodbrain barrier during HIV-1 encephalitis." J Immunol 158(7): 3499-3510.

Pialoux, G., S. Fournier, et al. (1997). "Central nervous system as a sanctuary for HIV-1 infection despite treatment with zidovudine, lamivudine and indinavir." Aids 11(10): 1302-1303.

Piliero, P. J. (2004). "Pharmacokinetic properties of nucleoside/nucleotide reverse transcriptase inhibitors." J Acquir Immune Defic Syndr 37 Suppl 1: S2-S12.

Pistell, P. J., S. Gupta, et al. (2010). "Metabolic and neurologic consequences of chronic lopinavir/ritonavir administration to C57BL/6 mice." Antiviral Res 88(3): 334-342.

Portegies, P., R. H. Enting, et al. (1993). "Presentation and course of AIDS dementia complex: 10 years of follow-up in Amsterdam, The Netherlands." AIDS 7(5): 669-675.

Powderly, W. G. (2010). "Integrase inhibitors in the treatment of HIV-1 infection." Journal of Antimicrobial Chemotherapy 65(12): 2485-2488.

Power, C., L. Boisse, et al. (2009). "NeuroAIDS: an evolving epidemic." Can J Neurol Sci 36(3): 285-295.

Price, R. W., B. Brew, et al. (1988). "The brain in AIDS: central nervous system HIV-1 infection and AIDS dementia complex." Science 239: 586-592.

Price, R. W., R. Parham, et al. (2008). "Enfuvirtide cerebrospinal fluid (CSF) pharmacokinetics and potential use in defining CSF HIV-1 origin." Antivir Ther 13(3): 369-374.

Prosperi, M. C., M. Zazzi, et al. (2010). "Low rate of virological failure and maintenance of susceptibility to HIV-1 protease inhibitors with first-line lopinavir/ritonavir-based antiretroviral treatment in clinical practice." J Med Virol 82(12): 1996-2003.

Qin, L., J. He, et al. (2008). "Increased systemic and brain cytokine production and neuroinflammation by endotoxin following ethanol treatment." J Neuroinflammation 5: 10.

Ragin, A. B., Y. Wu, et al. (2010). "Biomarkers of neurological status in HIV infection: a 3year study." Proteomics Clin Appl 4(3): 295-303. 
Ramirez, S. H., R. Potula, et al. (2009). "Methamphetamine disrupts blood-brain barrier function by induction of oxidative stress in brain endothelial cells." J Cereb Blood Flow Metab 29(12): 1933-1945.

Reynolds, A., C. Laurie, et al. (2007). "Oxidative stress and the pathogenesis of neurodegenerative disorders." Int Rev Neurobiol 82: 297-325.

Roberts, T. K., C. M. Buckner, et al. (2010). "Leukocyte transmigration across the blood-brain barrier: perspectives on neuroAIDS." Front Biosci 15: 478-536.

Roc, A. C., B. M. Ances, et al. (2007). Detection of Human Immunodeficiency Virus Induced Inflammation and Oxidative Stress in Lenticular Nuclei With Magnetic Resonance Spectroscopy Despite Antiretroviral Therapy. 64: 1249-1257.

Sacktor, N., N. Haughey, et al. (2004). "Novel markers of oxidative stress in actively progressive HIV dementia." J Neuroimmunol 157(1-2): 176-184.

Sacktor, N., G. Schifitto, et al. (2000). "Transdermal selegiline in HIV-associated cognitive impairment: pilot, placebo-controlled study." Neurology 54(1): 233-235.

Salminen, A., A. Kauppinen, et al. (2009). "ER stress in Alzheimer's disease: a novel neuronal trigger for inflammation and Alzheimer's pathology." J Neuroinflammation 6: 41.

Schifitto, G., B. A. Navia, et al. (2007). "Memantine and HIV-associated cognitive impairment: a neuropsychological and proton magnetic resonance spectroscopy study." Aids 21(14): 1877-1886.

Schifitto, G., C. T. Yiannoutsos, et al. (2009). "Selegiline and oxidative stress in HIVassociated cognitive impairment." Neurology 73(23): 1975-1981.

Schifitto, G., J. Zhang, et al. (2007). "A multicenter trial of selegiline transdermal system for HIV-associated cognitive impairment." Neurology 69(13): 1314-1321.

Sevigny, J. J., S. M. Albert, et al. (2004). "Evaluation of HIV RNA and markers of immune activation as predictors of HIV-associated dementia." Neurology 63(11): 2084-2090.

Shiu, C., E. Barbier, et al. (2007). "HIV-1 gp120 as well as alcohol affect blood-brain barrier permeability and stress fiber formation: involvement of reactive oxygen species." Alcohol Clin Exp Res 31(1): 130-137.

Sippy, B. D., F. M. Hofman, et al. (1995). "Increased expression of tumor necrosis factoralpha receptors in the brains of patients with AIDS." J Acquir Immune Defic Syndr Hum Retrovirol 10(5): 511-521.

Smurzynski, M., K. Wu, et al. (2011). "Effects of central nervous system antiretroviral penetration on cognitive functioning in the ALLRT cohort." Aids 25(3): 357-365 310.1097/QAD.1090b1013e32834171f32834178.

Smurzynski, M., K. Wu, et al. (2011). "Effects of central nervous system antiretroviral penetration on cognitive functioning in the ALLRT cohort." Aids 25(3): 357-365.

Soontornniyomkij, V., J. A. Nieto-Rodriguez, et al. (1998). "Brain HIV burden and length of survival after AIDS diagnosis." Clin Neuropathol 17(2): 95-99.

Steigbigel, R. T., D. A. Cooper, et al. (2010). "Long-term efficacy and safety of Raltegravir combined with optimized background therapy in treatment-experienced patients with drug-resistant HIV infection: week 96 results of the BENCHMRK 1 and 2 Phase III trials." Clin Infect Dis 50(4): 605-612.

Stephan, C., A. Carlebach, et al. (2007). "Dose reduction effective in alleviating symptoms of saquinavir toxicity." Int J STD AIDS 18(2): 81-84. 
Streck, E. L., G. K. Ferreira, et al. (2011). "Non-Nucleoside Reverse Transcriptase Inhibitors Efavirenz and Nevirapine Inhibit Cytochrome C Oxidase in Mouse Brain Regions." Neurochem Res.

Streck, E. L., G. Scaini, et al. (2008). "Effects of the HIV treatment drugs nevirapine and efavirenz on brain creatine kinase activity." Metab Brain Dis 23(4): 485-492.

Sturt, A. S., E. K. Dokubo, et al. (2010). "Antiretroviral therapy (ART) for treating HIV infection in ART-eligible pregnant women." Cochrane Database Syst $\operatorname{Rev(3):}$ CD008440.

Theys, K., K. Deforche, et al. (2010). "Estimating the individualized HIV-1 genetic barrier to resistance using a nelfinavir fitness landscape." BMC Bioinformatics 11: 409.

Touzet, O. and A. Philips (2010). "Resveratrol protects against protease inhibitor-induced reactive oxygen species production, reticulum stress and lipid raft perturbation." Aids 24(10): 1437-1447.

Tozzi, V., P. Balestra, et al. (2007). "Persistence of neuropsychologic deficits despite longterm highly active antiretroviral therapy in patients with HIV-related neurocognitive impairment: prevalence and risk factors." J Acquir Immune Defic Syndr 45(2): 174-182.

Tozzi, V., P. Balestra, et al. (2009). "Changes in Cognition During Antiretroviral Therapy: Comparison of 2 Different Ranking Systems to Measure Antiretroviral Drug Efficacy on HIV-Associated Neurocognitive Disorders." JAIDS Journal of Acquired Immune Deficiency $\quad$ Syndromes $\quad 52(1)$ 56-63 10.1097/QAI.1090b1013e3181af1083d1096.

Turner, J., L. Bansi, et al. (2010). "The prevalence of hepatitis C virus (HCV) infection in HIVpositive individuals in the UK - trends in HCV testing and the impact of $\mathrm{HCV}$ on HIV treatment outcomes." J Viral Hepat 17(8): 569-577.

Urquhart, B. L. and R. B. Kim (2009). "Blood-brain barrier transporters and response to CNSactive drugs." Eur J Clin Pharmacol 65(11): 1063-1070.

Van Den Bosch, L., P. Tilkin, et al. (2002). "Minocycline delays disease onset and mortality in a transgenic model of ALS." Neuroreport 13(8): 1067-1070.

Varghese, V., T. F. Liu, et al. (2010). "HIV-1 integrase sequence variability in antiretroviral naive patients and in triple-class experienced patients subsequently treated with raltegravir." AIDS Res Hum Retroviruses 26(12): 1323-1326.

Velliquette, R. A., T. O'Connor, et al. (2005). "Energy inhibition elevates beta-secretase levels and activity and is potentially amyloidogenic in APP transgenic mice: possible early events in Alzheimer's disease pathogenesis." J Neurosci 25(47): 10874-10883.

Walker, D. K., S. J. Bowers, et al. (2008). "Preclinical assessment of the distribution of maraviroc to potential human immunodeficiency virus (HIV) sanctuary sites in the central nervous system (CNS) and gut-associated lymphoid tissue (GALT)." Xenobiotica 38(10): 1330-1339.

Wang, H. and L. M. Tompkins (2008). "CYP2B6: new insights into a historically overlooked cytochrome P450 isozyme." Curr Drug Metab 9(7): 598-610.

Warnke, D., J. Barreto, et al. (2007). "Antiretroviral drugs." J Clin Pharmacol 47(12): 1570-1579.

Waters, L., M. Fisher, et al. (2011). "A phase IV, double-blind, multicentre, randomized, placebo-controlled, pilot study to assess the feasibility of switching individuals 
receiving efavirenz with continuing central nervous system adverse events to etravirine." Aids 25(1): 65-71.

Weiss, J., D. Theile, et al. (2007). "Inhibition of MRP1/ABCC1, MRP2/ABCC2, and MRP3/ABCC3 by nucleoside, nucleotide, and non-nucleoside reverse transcriptase inhibitors." Drug Metab Dispos 35(3): 340-344.

William, L. (2003). "Mitochondrial DNA replication, nucleoside reverse-transcriptase inhibitors, and AIDS cardiomyopathy." Progress in cardiovascular diseases 45(4): 305-318.

Winston, A., C. Duncombe, et al. (2010). "Does choice of combination antiretroviral therapy (cART) alter changes in cerebral function testing after 48 weeks in treatment-naive, HIV-1-infected individuals commencing cART? A randomized, controlled study." Clin Infect Dis 50(6): 920-929.

Winston, A., C. Duncombe, et al. (2010). "Does Choice of Combination Antiretroviral Therapy (cART) Alter Changes in Cerebral Function Testing after 48 Weeks in Treatment-Naive, HIV-1-Infected Individuals Commencing cART? A Randomized, Controlled Study." Clinical Infectious Diseases 50(6): 920-929.

Wolburg, H., S. Noell, et al. (2009). "Brain endothelial cells and the glio-vascular complex." Cell Tissue Res 335(1): 75-96.

Woods, S., D. Moore, et al. (2009). "Cognitive Neuropsychology of HIV-Associated Neurocognitive Disorders." Neuropsychology Review 19(2): 152-168.

$\mathrm{Xu}$, L. and M. C. Desai (2009). "Pharmacokinetic enhancers for HIV drugs." Curr Opin Investig Drugs 10(8): 775-786.

Yamamoto, B. K., A. Moszczynska, et al. (2010). "Amphetamine toxicities: classical and emerging mechanisms." Ann N Y Acad Sci 1187: 101-121.

Yeni, P., A. Mills, et al. (2010). "Etravirine in the treatment of HIV-1: a clinical overview for healthcare professionals." Curr HIV Res 8(7): 564-576.

Yilmaz, A., D. Fuchs, et al. (2006). "Cerebrospinal fluid HIV-1 RNA, intrathecal immunoactivation, and drug concentrations after treatment with a combination of saquinavir, nelfinavir, and two nucleoside analogues: the M61022 study." BMC Infectious Diseases 6(1): 63.

Yilmaz, A., M. Gisslen, et al. (2009). "Raltegravir cerebrospinal fluid concentrations in HIV-1 infection." PLoS One 4(9): e6877.

Yilmaz, A., R. W. Price, et al. (2008). "Persistent intrathecal immune activation in HIV-1infected individuals on antiretroviral therapy." J Acquir Immune Defic Syndr 47(2): 168-173.

Zembruski, N. C., G. Buchel, et al. (2011). "Potential of novel antiretrovirals to modulate expression and function of drug transporters in vitro." J Antimicrob Chemother 66(4): 802-812.

Zhong, Y., B. Hennig, et al. (2010). "Intact lipid rafts regulate HIV-1 Tat protein-induced activation of the Rho signaling and upregulation of P-glycoprotein in brain endothelial cells." J Cereb Blood Flow Metab 30(3): 522-533.

Zhou, H., E. C. Gurley, et al. (2006). "HIV protease inhibitors activate the unfolded protein response and disrupt lipid metabolism in primary hepatocytes." Am J Physiol Gastrointest Liver Physiol 291(6): G1071-1080.

Zink, M. C., J. Uhrlaub, et al. (2005). "Neuroprotective and anti-human immunodeficiency virus activity of minocycline." JAMA 293(16): 2003-2011. 


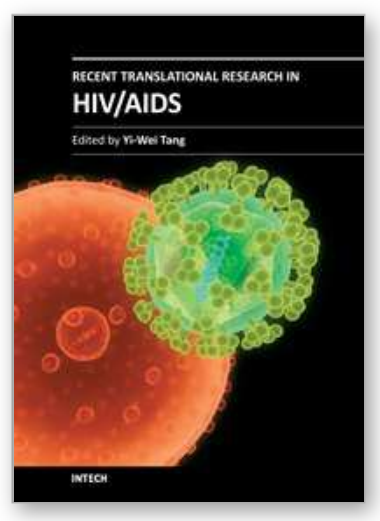

\author{
Recent Translational Research in HIV/AIDS \\ Edited by Prof. Yi-Wei Tang
}

ISBN 978-953-307-719-2

Hard cover, 564 pages

Publisher InTech

Published online 02, November, 2011

Published in print edition November, 2011

The collective efforts of HIV/AIDS research scientists from over 16 countries in the world are included in the book. This 27-chapter Open Access book well covers HIV/AIDS translational researches on pathogenesis, diagnosis, treatment, prevention, and also those beyond conventional fields. These are by no means inclusive, but they do offer a good foundation for the development of clinical patient care. The translational model forms the basis for progressing HIV/AIDS clinical research. When linked to the care of the patients, translational researches should result in a direct benefit for HIV/AIDS patients.

\title{
How to reference
}

In order to correctly reference this scholarly work, feel free to copy and paste the following:

Cagla Akay and Kelly L. Jordan-Sciutto (2011). Antiretroviral Therapy and HIV-Associated Neurocognitive Disorders, Recent Translational Research in HIV/AIDS, Prof. Yi-Wei Tang (Ed.), ISBN: 978-953-307-719-2, InTech, Available from: http://www.intechopen.com/books/recent-translational-research-in-hivaids/antiretroviral-therapy-and-hiv-associated-neurocognitive-disorders

\section{INTECH}

open science | open minds

\section{InTech Europe}

University Campus STeP Ri

Slavka Krautzeka 83/A

51000 Rijeka, Croatia

Phone: +385 (51) 770447

Fax: +385 (51) 686166

www.intechopen.com

\section{InTech China}

Unit 405, Office Block, Hotel Equatorial Shanghai

No.65, Yan An Road (West), Shanghai, 200040, China

中国上海市延安西路65号上海国际贵都大饭店办公楼 405 单元

Phone: +86-21-62489820

Fax: +86-21-62489821 
(C) 2011 The Author(s). Licensee IntechOpen. This is an open access article distributed under the terms of the Creative Commons Attribution 3.0 License, which permits unrestricted use, distribution, and reproduction in any medium, provided the original work is properly cited. 